\title{
Is mycobacterial vaccination a risk for septic arthritis in pediatric age? A Case report
}

\author{
Chico-Carpizo Fernando ${ }^{1}$, Colmenares-Bonilla Douglas ${ }^{2}$, Casimiro Guzman Leticia ${ }^{3}$, Leon Espitia Jose ${ }^{4}$, Osorio Guzman Monica ${ }^{5}$, Ange \\ Cruz Luis ${ }^{6}$, Dominguez Gasca Luis Gerardo ${ }^{7}$ and Tovar-Cardona Mayra ${ }^{8}$ \\ ${ }^{1}$ Second year resident in Traumatology and orthopedics. Division of Surgery at Leon General Hospital, Leon, Guanajuato, Mexico \\ ${ }^{2}$ Musculoskeletal Division, Pediatric Orthopedic Department, Hospital Regional de Alta Especialidad del Bajio, Leon, Guanajuato, Mexico \\ ${ }^{3}$ Anatomopathologist, General Hospital. Leon, Guanajuato, Mexico \\ ${ }^{4}$ Paediatric Oncology, General Hospital, Leon, Guanajuato, Mexico \\ ${ }^{5}$ Paediatric Infectology, General Hospital, Leon, Guanajuato, Mexico \\ ${ }^{6}$ Paediatric Orthopedic Surgeon, Division of Surgery; General Hospital, Leon, Guanajuato, Mexico \\ ${ }^{7}$ Third year resident in Traumatology and orthopedics, Division of Surgery at Leon General Hospital; Leon, Guanajuato, Mexico \\ ${ }^{8}$ Paediatric Orthopedic Fellow, Hospital Regional de Alta Especialidad del Bajio; Leon, Guanajuato, Mexico
}

\begin{abstract}
Background: Tuberculosis continues to be a global health problem, being one of the first 10 causes of death worldwide and among the first due to infectious disease. In 2015, 1 million new cases were reported around the world, only in children, accounting $10 \%$ of the total. Bone infection due to mycobacteria comprises only $10 \%$ of cases of musculoskeletal tuberculosis and $1 \%$ to $2 \%$ of cases of tuberculosis in general. The condition of long bones represents only $14 \%$. Mycobacterium Bovis infection accounts for about $1 \%$ of infections with the Mycobacterium Tuberculosis complex. Only $5 \%$ of infections with $M$. Bovis have affection to bone or joints. Suspect $M$. Bovis after BCG (0.39 per million vaccinations), which has a predilection for metaphysis and epiphysis of long bones, with onset of symptoms from 1 to 1.5 years after vaccination and radiographic pattern similar to that of the patient in this case.
\end{abstract}

Objective: to raise awareness and focus on the importance of approaching bone lesions, considering bone tuberculosis among differential diagnoses, for an early diagnosis.

Material and methods: A case description study is performed, reporting a 20 months old male, with bone pain and lesion in the proximal tibia, the study and treatment of the patient is described, as well as functional results. Results: Follow-up at 6 months with adequate evolution and response to an antitumor treatment. Last radiograph of control with classification of grade III, according to the Montoya classification, with adequate functional evolution, classification 6 according to the scale of functional mobility.

Conclusions: Importance of timely diagnosis in this type of injuries, to provide adequate treatment and improve prognosis in the short, medium and long term.

\section{Introduction}

Tuberculosis continues to be a public health problem worldwide, in 2015 there were 10.4 million new cases reported, 1 million of these affect children. With appropriate treatment and prevention, incidence has been reduced, but without achieving the proposed goals, there are still important faults in early diagnosis and treatment [1].

Pulmonary tuberculosis comprises clear majority of cases. According to a study conducted in Europe from 2002 to 2011 which evaluates extrapulmonary manifestations, it reports that tuberculosis comprises around $19 \%$ in adults and 18 to $37 \%$ in children younger than 14 years. Within extrapulmonary tuberculosis, bone disease accounts for about $4 \%$, with the spine being the most frequent site of involvement (about 35\% to 50\% of cases). The involvement of long bones represents only $14 \%$ of cases of musculoskeletal involvement, with intra-articular or tuberculous arthritis being more frequent than long bone involvement [2-8].

The infection by non-tuberculous mycobacteria, are even less frequent. Importing from $1-10 \%$ of the total of those caused by the
Mycobacterium tuberculosis complex, are the infections caused by Mycobacterium Bovis, only 5\% have affection to bone or joints. The main forms of transmission are respiratory and enteral. A study by Córdova et al. in 2012 in Buenos Aires, it reported a frequency of $0.4 \%$ isolation [9-13]. In Mexico, a study was carried out by Lopez-Alvarez et al. in 2010, and reported isolation of less than $1 \%$ of M. Bovis [14]. Currently, up to $26 \%$ isolation of M. Bovis is reported. In Mexico, up to $16.5 \%$ of bovine tuberculosis is reported in dairy products, as up to $30 \%$ of these are sold without pasteurization, producing an important infectious source in our environment. M. Bovis has shown antimicrobial resistance of up to $15 \%$ for streptomycin, $9 \%$ for isoniazid and 3\% for rifampicin, in addition to natural resistance to pyrazinamide, in the

${ }^{*}$ Correspondence to: Douglas Colmenares Bonilla, Musculoskeletal Division, Pediatric Orthopedic Department, Hospital Regional de Alta Especialidad del Bajio, Leon, Guanajuato, Mexico, E-mail: douglas_cb@yahoo.com

Key words: Tuberculous osteomyelitis, $M$. Bovis, BCG

Received: August 01, 2018; Accepted: August 13, 2018; Published: August 20, 2018 
samples in which it was isolated, in the study conducted by Bobadilla del Valle and colleagues in 2015 [3,5].

The Bacillus Calmette-Guerin (BCG) vaccine has been used for the prevention of tuberculosis since 1921 [10]. In countries where it is used, coverage of up to $90 \%$ is reported. In most children, the inoculation of BCG is not harmful. There have been reports of infection due to the BCG vaccine since 1970 in different studies where the incidence varies from 1.7 to 10.1 in the last reports [4]. Risk factors include the type and dose of the vaccine. This condition usually affects the peripheral skeleton, the most common sites of affection are metaphysis and epiphysis of long bones, with involvement of the legs in up to $58 \%$ of the cases, this differs with the usual presentation of tuberculous osteomyelitis, which has a preference for the spine and weight bearing joints, with an age preference in adolescents and school children. The onset of symptoms varies from 1-1.5 to 5 years $[4,10]$.

\section{Case Report}

A 20 months toddler starts with claudication and pain in the right leg for more than two months. He suffers pain in the left knee that remains day and night, even preventing him from walking.

In his initial assessment with a family doctor, he is prescribed analgesic and rest at home.

There is no history of personal or family illness. Family members do not remember a history of trauma or recurrent infections. There is also no data to suggest a tumor.

The only suspected history is the usual vaccination of BCG and the regular consumption of direct dairy products from the barn.

After two weeks, and having no improvement in the picture, he is taken to the second hospital, where he enters and starts the approach with laboratory studies.

The child is observed with weight and height according to his age without attack to the general state. There is increased volume in the anterior region of the leg and pain when playing, running and touching the skin on the right knee. An episode of fever not quantified for three days before, controlled with paracetamol, without repeating.

Laboratory studies were taken, 261 alkaline phosphatase, 815 lactic dehydrogenase, other normal studies were reported (Table 1).

In the radiographs, lytic lesion of $3 \times 3 \mathrm{~cm}$ was observed in the proximal medial metaphysis of the right tibia, destruction of the medial cortex, without involvement of the soft tissues or periosteal reaction (Figure 1). Under the suspicion of a malignant tumor, X-ray studies are completed, without finding other lesions. The protocol is concluded with biopsy of the tumor.

Surgical approach described an infiltrative lesion of bone, welldefined borders, sized $30 \times 30 \mathrm{~mm}$ with caseous, brown, necrotic content. Thickened periosteum and fibrous consistency. After draining the lesion, it is then irrigated with saline solution, subsequently repairing the periosteum. The extremity was immobilized with splint for three weeks.

The sample is studied by means of immunohistochemistry on suspicion of histiocytosis $\mathrm{x}$, with negative result. The Ziehl-Nielsen stain and Polymerase chain reaction (PCR) reports positivity for Mycobacterium bovis. The patient started treatment with Isoniazid, Rifampin, Ethambutol and Pyrazinamide for two months, followed by ten months of Isoniazid, Rifampin.
Table 1. Laboratory studies on admission of the patient to hospitalization for study

\begin{tabular}{|l|c|c|}
\hline Blood Exams & Patient & Normal ranges \\
\hline Hemoglobin & 12.5 & $12-16$ \\
\hline Hematocrit & 36.7 & $35-54$ \\
\hline Platelets & 350 & $150-450$ \\
\hline Lekocytes & 13.43 & $6-17$ \\
\hline Neutrophils & $52.2 \%$ & $37-72 \%$ \\
\hline Lymphocytes & $37.9 \%$ & $20-38 \%$ \\
\hline Alkaline Phosphatase & 261 & $40-129$ \\
\hline Lactic Deshidrogenase & 815 & $313-618$ \\
\hline Glucose & 114 & $75-110$ \\
\hline
\end{tabular}

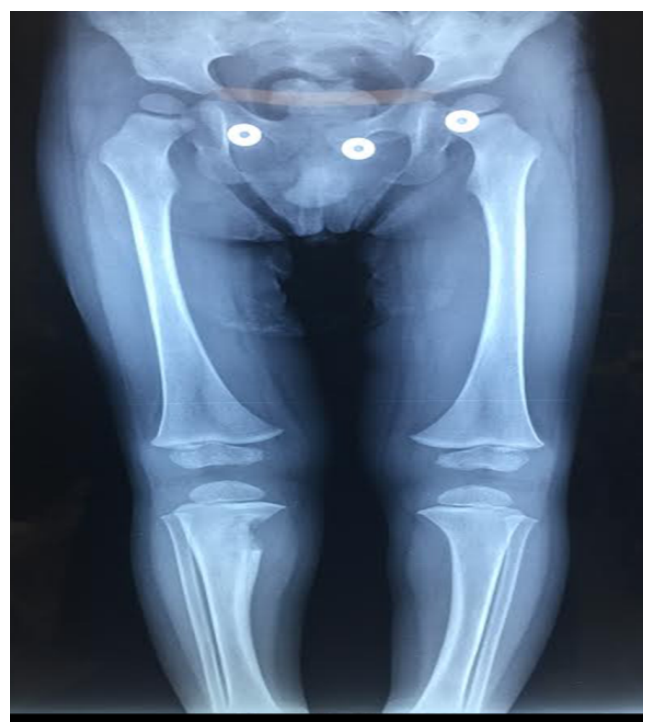

Figure 1. Image showing a lytic lesion in the proximal region of the tibia, with ill-defined borders

\section{Follow up}

The 18-month evolution does not suggest local or distant recurrence, with response to treatment. The follow-up was performed with clinical assessment of mobility arcs, as well as radiographic images in search of consolidation and / or recurrence of local or distant disease. On examination with full knee mobility arches at $120^{\circ}$ flexion and $0^{\circ}$ extension. Twelve months' follow up x rays showed full consolidation in bone defect (Figure 2), without angular deformity or suggested physeal damage, the surgical wound scar is without complications (Figure 3), and grade 6 according to the Functional motor scale, performing normal activities for his age without limitations.

\section{Discussion}

The cases of bone infection due to non-tuberculous mycobacteria without joint involvement are very rare, making diagnosis difficult. Tuberculous osteomyelitis simulates chronic bacterial osteomyelitis, tumors or granulomatous lesions [15], so the index of suspicion should be stimulated to achieve early diagnosis.

The transmission of Mycobacterium Bovis is generally enteral, respiratory or as a complication by the application of BCG. In our environment, the isolation figure has increased considerably in recent years, hence the importance of considering it as a differential diagnosis in this type of bone lesions [3].

In the study by Vohra et al. they analyzed 28 lesions in 25 patients between the years 1988 and 1995, with an average age of 26 years, 


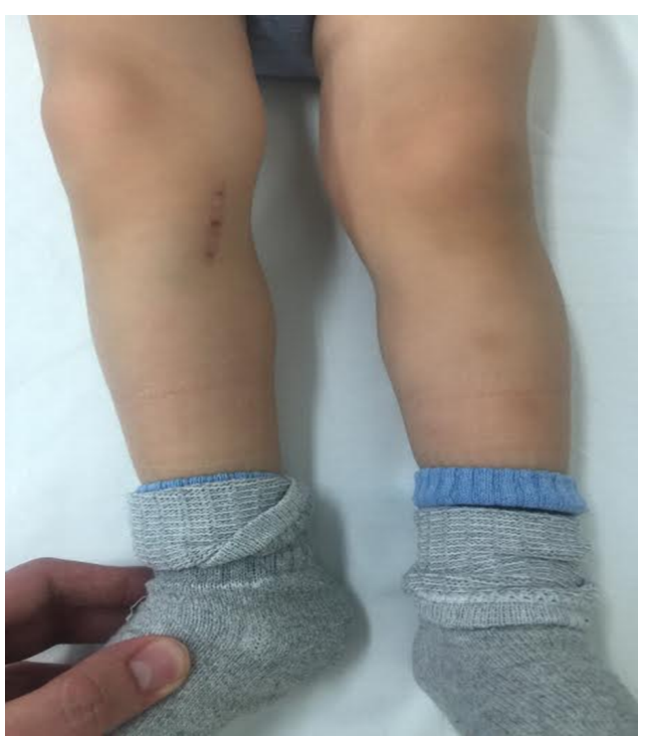

Figure 2. Clinical aspect of the patient two weeks after the biopsy and curettage of the lesion

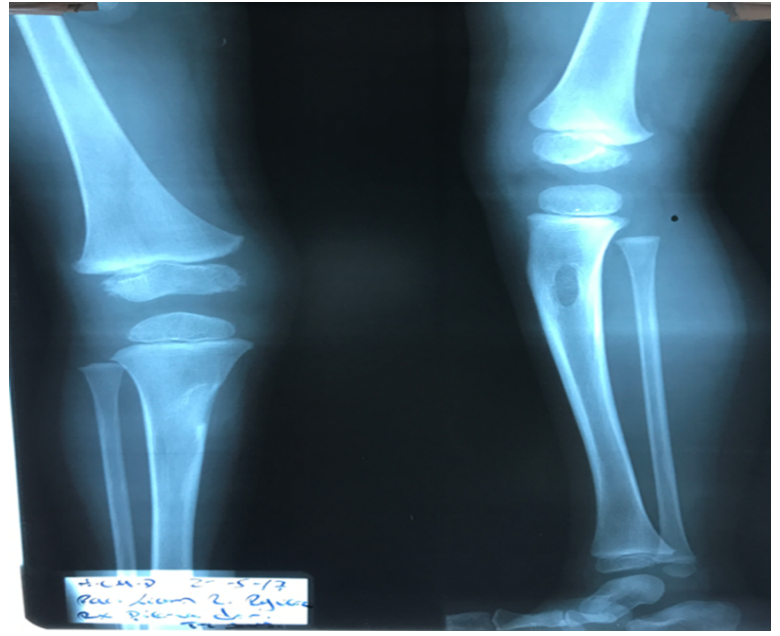

Figure 3. Radiography 18 months of follow-up, observing the response to treatment and recovery of bone anatomy

$40 \%$ were in lower limbs, without reporting cases in the tibia. Six patients with lesions in other sites and six others with associated lung infection. Pain and swelling were the most frequent symptoms, as in our patient. Only $32 \%$ presented radiographic images characteristic of bone tuberculosis, the rest with characteristics of chronic pyogenic osteomyelitis or neoplasms. In $88 \%$ of the patients, the diagnosis was confirmed by histopathology. The average follow-up was 41 months, with symptom improvement in all patients at six months. In all cases, there was improvement in bone lesions during follow-up and more than $90 \%$ of patients with complete and asymptomatic mobility arches at the end of treatment [15].

Aparicio et al. report a retrospective study of four patients with osteoarticular tuberculosis between the years of 2008 and 2010. Of the four cases, there were two knee arthritis, one wrist involvement and one tenosynovitis of the left anterior tibialis. All were adults between 33 and 76 years old. All the patients presented repeated episodes of pain and swelling of the affected joint. On radiographs, the patient with a wrist condition had great bone destruction. Only one of the patients evidenced lung disease. Only one of the cases presented positive bacilloscopy and two cases with positive Mantoux test. In two cases
M. tuberculosis was isolated, in another M. africanum, and in one of the cases mycobacteria were not isolated, but the Mantoux test and the response to treatment confirmed the diagnosis. All patients were treated with the 4 antimicrobial agents for 2 months and 4 months with isoniazid and rifampin. The follow-up was 2 years on average, all patients evolved to cure. The functional result was excellent for the two patients with knee involvement; bad for the patient of the wrist that ended with ankylosis, and that of the anterior tibial synovitis, presented a secondary epidural abscess with affection of the flexion and extension of the ankle [8].

In 2015, in Canada, Khan et al. documented the case of a 2-yearold infant with a clinical picture of progressive claudication, tenderness and increased heel volume. The patient born in India and Canadian resident, with a similar clinical course than present case, who was treated with cefazolin, evolving unfavorably with fistula and abscess, so after confirming the tuberculous infection he was given specific treatment. Being resistant to pyrazinamide, $M$. bovis as a causative agent was suspected, the management is ajusted, improving clinically, which accentuates the need for adequate diagnosis [10].

In 2014 Yoo et al. reviewed eight cases of mycobacterial osteomyelitis with epiphyseal involvement in healthy infants, with an age range of 12 to 25 months, four cases were positive for Mycobacterium Bovis BCG, Mycobacterium tuberculosis in three cases, and another for nontuberculous mycobacteria. In six of the cases, the lesion was found in the distal femoral epiphyseal region, one case in the proximal tibia and another in the proximal humerus. Six of the patients manifested as chronic osteomyelitis with pain or limitation of mobility; the other two patients had acute onset osteomyelitis with greater general affection, unlike the other reports, no alterations were found in blood studies. In all cases without improvement despite antibiotic treatment, which leads to the suspicion of mycobacterial infection [15].

\section{Conclusions}

In the present study, although it is the report of an isolated case, it leads us to raise awareness and focus on the approach of patients with this type of bone lesions. The clinical presentation of mycobacterial infection is nonspecific and insidious, and the diagnosis is not simple, worsening the prognosis in the short, medium and long term. As has been demonstrated, in our region, the incidence of mycobacterial infection is high [3], representing a public health problem. It should be kept in mind as a differential diagnosis in this type of lesions, for an adequate diagnosis and timely treatment.

\section{References}

1. Global tuberculosis report (2017) Geneva: World Health Organization; 2017. Licence CC BY-NCSA.

2. Sandgren A, Hollo V, van der Werf MJ (2013) Extrapulmonary tuberculosis in the European Union and European Economic Area, 2002 to 2011. Euro Surveill.

3. Bobadilla-del Valle M, Torres-González P, Cervera-Hernández ME, Martínez-Gamboa A, Crabtree-Ramirez B, et al. (2015) Trends of Mycobacterium Bovis Isolation and First-Line Anti-tuberculosis Drug Susceptibility Profile: A Fifteen-Year LaboratoryBased Surveillance. PLoS Negl Trop Dis 9: e0004124. [Crossref]

4. Kröger L, Brander E, Korppi M, Wasz-Höckert O, Backman A, et al. (1994) Osteitis after newborn vaccination with three different Bacillus Calmette-Guerin vaccines: twenty-nine years of experience. Pediatr Infect Dis J 13: 113-6. [Crossref]

5. Hlavsa MC, Moonan PK, Cowan LS, Navin TR, Kammerer JS, et al. (2008) Human tuberculosis due to Mycobacterium bovis in the United States, 1995-2005. Clin Infect Dis 47: 168-175. [Crossref]

6. American Academy of Pediatrics (2015) Red Book 2015, Report of the Committee of Infectious Diseases, 30th edition, 805-836. 
7. Pérez Durán MJ, Moreno Sanz-Gadea B, del Rosal Raves T, Mellado Peña MJ, Baquero-Artigao F (2017) Osteoarticular tuberculosis in paediatrics: A review of 20 years of cases in a tertiary hospital. An Pediatr (Barc) 87: 291-292. [Crossref]

8. Aparicio G, Viudez I, Pérez JL, Delgado F (2012) Extrapulmonary and extraspinal presentation of osteoarticular tuberculosis. Acta Ortop Mex 26: 15-20. [Crossref]

9. Cañabate Reche F, Daza Torres A, Campos Aguilera A, López Prieto F, Vázquez López M, et al. (1997) Osteoarticular tuberculosis during infancy: the reality in our environment. An Esp Pediatr 46: 73-6. [Crossref]

10. Khan S, Stimec J, Kitai I (2015) Nonresponding osteomyelitis in a two-year-old boy. CMAJ 187: 901-904. [Crossref]

11. Lin CJ, Yang WS, Yan JJ, Liu CC (1999) Mycobacterium bovis osteomyelitis as a complication of Bacille Calmette-Guérin (BCG) vaccination: rapid diagnosis with use of DNA sequencing analysis: a case report. J Bone Joint Surg Am 81: 1305-11. [Crossref]
12. Yoo WJ, Choi IH, Yun YH, Cho TJ, Cheon JE, et al. (2014) Primary Epiphyseal Osteomyelitis Caused by Mycobacterium Species in Otherwise Healthy Toddlers. $J$ Bone Joint Surg Am 96: e145. [Crossref]

13. Cordova E, Gonzalo X, Boschi A, Lossa M, Robles M, et al. (2012) Human Mycobacterium bovis infection in Buenos Aires: epidemiology, microbiology and clinical presentation. Int J Tuberc Lung Dis 16: 415-417. [Crossref]

14. Lopez-Alvarez R, Badillo-Lopez C, Cerna-Cortes JF, Castillo-Ramirez I, RiveraGutierrez S, et al. (2010) First insights into the genetic diversity of Mycobacterium tuberculosis isolates from HIV-infected Mexican patients and mutations causing multidrug resistance. BMC Microbiol 10: 82. [Crossref]

15. Vohra R, Kang HS, Dogra S, Saggar RR, Sharma R (1997) Tuberculous osteomyelitis. J Bone Joint Surg Br 79: 562-6. [Crossref]

Copyright: $\mathbb{0} 2018$ Chico-Carpizo F. This is an open-access article distributed under the terms of the Creative Commons Attribution License, which permits unrestricted use, distribution, and reproduction in any medium, provided the original author and source are credited. 